\title{
ANÁLISE SENSORIAL DE COOKIE DE AVEIA, MEL E CASCA DE BANANA EM COMENSAIS DE UM RESTAURANTE COMERCIAL DE GUARAPUAVA-PR
}

\author{
SENSORY ANALYSIS OF OATMEAL, HONEY AND BANANA PEEL COOKIE IN \\ INMATE'S COMMERCIAL RESTAURANT DINERS OF GUARAPUAVA, PR
}

\author{
Luane Aparecida AMARAL"; Brenda Alana RIBAS²; Raquel Rosalva GATTI³ Renata \\ Leia DEMARIO4; Kélin SCHWARZ5; Priscilla Negrão de MOURA ${ }^{6}$
}

1 - Nutricionista. Mestranda do Programa de Pós Graduação em Saúde e Desenvolvimento na Região Centro Oeste da Universidade Federal de Mato Grosso do Sul

2 - Nutricionista. Universidade Estadual do Centro-Oeste (UNICENTRO), Guarapuava, PR.

3 - Nutricionista, Doutora em Ciências da Educação, Docente do Departamento de Nutrição da Universidade Estadual do Centro-Oeste (UNICENTRO), Guarapuava, PR.

4 - Nutricionista, Mestre em Nutrição, Docente do Departamento de Nutrição da Universidade Estadual do Centro-Oeste (UNICENTRO), Guarapuava, PR.

5 - Nutricionista, Doutora em Ciências (Energia Nuclear na Agricultura e no Ambiente), Docente do Departamento de Nutrição da Universidade Estadual do Centro-Oeste (UNICENTRO), Guarapuava, PR.

6 - Nutricionista, Doutora em Medicina Interna, Docente do Departamento de Nutrição da Universidade Estadual do Centro-Oeste (UNICENTRO), Guarapuava, PR.

Autor para correspondência: luapamaral@hotmail.com

\section{RESUMO:}

A mudança na sociedade contemporânea têm colaborado para o aumento de refeições realizadas fora do domicílio, demandando mais Unidades Produtoras de Refeições que por sua vez geram muitos resíduos orgânicos. Com isso este estudo objetivou avaliar a aceitabilidade sensorial de cookies de banana com e sem casca afim de auxiliar no reaproveitamento de alimentos. Participaram da pesquisa 50 provadores não treinados, sendo adultos que frequentavam um restaurante comercial na cidade de Guarapuava, PR, de ambos os gêneros, com idade entre 18 a 60 anos. As amostras foram submetidas a análise sensorial, sendo avaliados os atributos de aparência, aroma, sabor, textura, cor e aceitação global, através de uma escala hedônica. Os indivíduos receberam uma porção de cada amostra (aproximadamente $10 \mathrm{~g}$ ). Foram elaboradas duas formulações de cookies de aveia e mel, F1 - com adição de casca de banana e F2 com banana. Em relação à aparência, aroma, textura e cor não houve diferenças significativas entre as amostras. Para sabor e sabor residual houve diferenças significativas de 5\%. A amostra F2 obteve maior aceitabilidade e intenção de compra, comparados a F1, porém, apenas para a intenção de compra houve diferença significativa de 5\%. Aproximadamente $60 \%$ dos provadores preferiram a amostra F2. Os cookies com adição de casca de banana foram bem aceitos entre os comensais, sendo considerados uma boa alternativa para a utilização das partes não convencionais e para a comercialização por ser um produto diferenciado e rico em fibras.

Palavras-chave: Alimentação Coletiva, Alimento, Aproveitamento Integral dos Alimentos

\section{ABSTRACT:}

The change in contemporary society has contributed to the increase of meals performed outside the home, demanding more units to produce meals that in turn generate a lot of 
organic waste. This study aimed to evaluate the sensory acceptability of banana cookies with and without peel in order to assist in the reuse of food. Participated in the survey 50 tasters untrained. Being adults who attended a commercial restaurant in the city of Guarapuava, PR, of both genders, aged between 18 and 60 year. Samples were submitted to sensory analysis, being evaluated the appearance attributes, aroma, flavor, texture, color and overall acceptance, through a hedonic scale. The individuals received a portion of each sample (10 g Approximately). Were elaborate two formulations of oatmeal cookies and honey, F1 - with added banana peel and F2 with banana. Results: in relation to appearance, aroma, texture and color there were no significant differences between the samples. To taste and aftertaste were significant differences of $5 \%$. The F2 sample obtained larger acceptability and intent to purchase, compared to $\mathrm{F} 1$, however, only purchase intent were significant difference of $5 \%$. Approximate $60 \%$ of the tasters liked more sample F2. Conclusion: The cookies with added banana peel were well accepted among diners, being considered a good alternative to the use of non-conventional parts and for marketing to be a differentiated product and rich in fiber.

Keywords: Collective Feeding, Food, Whole Utilization of Foods.

\section{INTRODUÇÃO}

O padrão alimentar atual da sociedade é caracterizado pela busca de refeições fora de casa devido à falta de tempo para o preparo e consumo no próprio lar. Portanto, observase como consequência o aumento do número de Unidades Produtoras de Refeições (UPRs) para atender esta população. (AKUTSU, et al., 2005)

A produção de alimentos gera diversos resíduos orgânicos e inorgânicos (Santos; Simões; Martens; 2006). Portanto, é necessário que as UPRs adquiram práticas que preservem os recursos naturais e minimizem os danos ao ambiente (GARCIA, 2003; COSTELLO, et al., 2009; FRIEL, et al., 2009). Nesse contexto, estudos estão surgindo com a adição de cascas de frutas e vegetais em diversas preparações obtendo boa aceitação sensorial. (SILVA; SILVA, 2012; REIS, et al., 2013).

Os resíduos como as cascas dos alimentos apresentam na maioria das vezes concentrações mais elevadas de nutrientes do que a sua parte comestível. Portanto, é importante a sua utilização, pois o aproveitamento integral dos alimentos, além de minimizar os gastos com alimentação e melhorar a qualidade nutricional do cardápio, diminui 0 desperdício de alimentos e possibilita a criação de novas receitas, como, por exemplo, sucos, doces, e geleias. (GONDIN, et al., 2005).

Um exemplo de aproveitamento de alimentos é a utilização da casca da banana que é considerada uma das frutas de maior consumo em todo o mundo e definida como um alimento energético composto por fibras, vitaminas e na sua maior parte por minerais 
(EMBRAPA, 1997). Alguns estudos mostram que na sua casca, encontra-se as pectinas que são fibras responsáveis pela formação de géis que servem para o enriquecimento de produtos alimentícios. (EMAGAa, et al., 2008, EMAGAb, et al., 2008; MANEERAT; TANGSUPHOOM; NITITHAMYONG, 2017).

Maneerat, Tangsuphoom e Nitithamyong (2017) extraíram a pectina de cascas de banana e utilizaram como substituto da gordura em creme e obtiveram bons resultados, sendo uma alternativa como substituto de gordura em produtos alimentares.

Carvalho e Conti-Silva (2017) utilizaram as cascas de banana em barras de cereais e observaram boa aceitabilidade, mesmo em diferentes concentrações, o que pode favorecer o desenvolvimento de novos produtos. Outro estudo utilizou as cascas de banana na fabricação de doces, e seus resultados indicaram que a formulação de $20 \%$ de cascas obteve boa aceitação e alta quantidade de fibras dietéticas. (OLIVEIRA, et al., 2009).

Visando a importância do reaproveitamento dos resíduos que seriam descartados no meio ambiente e com o intuito de redução de custos e melhora da qualidade nutricional dos mesmos, o objetivo do estudo foi avaliar a aceitabilidade sensorial de cookies de banana com e sem casca de banana.

\section{METODOLOGIA}

\subsection{Obtenção de matéria prima}

Os ingredientes foram adquiridos em um supermercado local do Município de Guarapuava-Pr. Utilizou-se para a pesquisa bananas tipo caturra, maduras, com coloração amarela escura uniforme, ou seja, sem manchas ou imperfeições.

\subsection{Formulação}

Foram elaboradas duas formulações de cookies de aveia e mel, F1 - com adição de casca de banana e F2 com banana. Na Tabela 1, podem ser observados os ingredientes utilizados na formulação dos cookies.

O preparo das amostras ocorreu no laboratório de Técnica Dietética da Universidade Estadual do Centro Oeste - UNICENTRO em Guarapuava-PR 24 horas antes 
do teste de aceitabilidade. Todos os ingredientes foram pesados em balança Mondial.

O cookie de casca de banana foi elaborado da seguinte forma: foi retirada as cascas das bananas e higienizadas em água corrente e mantidas em solução de água clorada (10 $\mathrm{ml}$ de água sanitária para uso geral e 1 litro de água) durante 15 minutos. Posteriormente as cascas foram cozidas à $100^{\circ} \mathrm{C}$ juntamente em água e mel durante 15 minutos. (ANVISA, 1999).

As cascas foram trituradas em liquidificador doméstico até obter aspecto homogêneo. Em seguida foi adicionado aos demais ingredientes mexendo até formar uma massa homogênea, para decorar foi adicionado a massa chocolate meio amargo e dispostos em formas, levado ao forno à $180 \stackrel{\circ}{\mathrm{C}}$ por 20 minutos. $\mathrm{O}$ mesmo ocorreu com $\mathrm{O}$ cookie com a banana.

\subsection{Análise sensorial}

Participaram da pesquisa 50 provadores não treinados, sendo adultos que frequentavam um restaurante comercial na cidade de Guarapuava, PR, de ambos os gêneros, com idade entre 18 a 60 anos.

Os participantes foram abordados na entrada do restaurante no horário do almoço. As amostras foram submetidas à análise sensorial na praça de alimentação. Cada prova foi feita individualmente, sendo que o provador foi orientado pelas pesquisadoras para o preenchimento das respostas.

Foram avaliados os atributos de aparência, aroma, sabor, textura, cor e aceitação global, através de uma escala hedônica estruturada de 9 pontos, variando de 1 ("desgostei muitíssimo") a 9 (gostei muitíssimo).

Para avaliar a intenção de compra foi utilizado uma escala hedônica de 5 pontos variando de 1 ("certamente não compraria") a 5 ("certamente compraria").

Os provadores também responderam qual amostra gostaram mais.

Os julgadores receberam uma porção de cada amostra (aproximadamente $10 \mathrm{~g}$ ), em copos descartáveis, acompanhados de água para realização do branco. As formulações foram oferecidas aos julgadores de forma monádica sequencial.

\section{4 Índice de aceitabilidade (IA)}

O cálculo do IA das formulações foi realizado segundo a fórmula: IA (\%) = A x 100/B 
( $\mathrm{A}$ = nota média obtida para o produto; $\mathrm{B}=$ nota máxima dada ao produto). (DUTCOSKI, 2011).

\subsection{Análise Estatística}

Os dados foram analisados com auxílio do software ASSISTAT®, versão 7.7, através da análise de variância (ANOVA). A comparação de médias foi realizada pelo teste de médias de Tukey.

\subsection{Questões éticas}

Este trabalho foi aprovado pelo Comitê de Ética em Pesquisa da UNICENTRO, parecer número ํㅜ 840.222. Entretanto, como critérios de exclusão foram considerados os seguintes fatores: menores de 18 anos, maiores de 60 anos, possuir alergia a algum ingrediente utilizado na elaboração do brigadeiro e não entregar o Termo de Consentimento Livre e Esclarecido (TCLE) assinado.

\section{RESULTADOS E DISCUSSÃO}

Os provadores tinham média de $37,95 \pm 13,47$ anos e $62 \%(n=31)$ eram do sexo feminino. O IA e as médias para os atributos: aparência, aroma, sabor, sabor residual, textura, cor, aceitação global e intenção de compra estão dispostos na Tabela 2.

Em relação à aparência, aroma, textura e cor não houve diferenças significativas entre as amostras, o mesmo foi encontrado por Carvalho e Conti-Silva (2017) ao avaliarem barras de cereais com casca de banana. Para sabor e sabor residual houve diferenças significativas de $5 \%$.

Os cookies com casca de banana obtiveram uma menor nota no quesito cor e sabor residual. O escurecimento deve-se à hidrólise de polissacarídeos presentes na casca e açúcares adicionados ao cookie desencadeando a reação de Maillard além da caramelização de açúcares devido ao aquecimento (BOBBIO; BOBBIO, 2001). O mesmo foi encontrado por Santos (2014) ao avaliar a aceitação de bananada com a casca da banana. O leve sabor residual, foi encontrado também por Miranda et al., (2013) que verificou a aceitabilidade de bolos enriquecidos com farinha de casca de maracujá. 
A amostra F2 obteve notas maiores nos atributos aceitação global e intenção de compra, comparados a F1, porém, apenas para a intenção de compra houve diferença significativa de $5 \%$.

De acordo com vários estudos, para que um produto seja considerado aceito, de acordo com os aspectos sensoriais, é necessário que o Índice de Aceitabilidade (IC) seja de no mínimo 70\%. Logo, neste estudo as duas amostras foram bem aceitas evidenciando que podem ser comercializadas (Tabela 2). (ISHIMOTO, et al., 2007; MIGUEL et al., 2008; BRAGA, et al., 2009).

\section{CONCLUSÃO}

Os cookies com adição de casca de banana foram aceitos entre os comensais, porém o padrão obteve maiores notas. A alternativa de se utilizar partes não convencionais em preparações é válida afim de testar novos produtos, enriquecido com fibras dietéticas. Sugere-se mais estudos com casca de banana para avaliar seu potencial na indústria e possíveis métodos que minimizem o escurecimento e sabor residual nesses produtos.

\section{REFERÊNCIAS}

AKUTSU, R.C.; BOtelho, R. A.; CAMARGO, E. B.; SÁVIO, K. E. O.; ARAÚJO, W. C. Adequação das Boas práticas de fabricação em serviços de alimentação. Revista de Nutrição, v. 18, n. 3, p. 419-27, 2005.

SANTOS CM, SIMÕES JC, MARTENS SH. O gerenciamento de resíduos sólidos no curso superior de tecnologia em gastronomia. Nutrição em Pauta, v. 14, n. 77, p. 44-9, 2006.

COSTELLO, A.; ABBAS, M.; ALLEN, A.; BALL, S.; BELL, S.; BELLAMY, S.; FRIEL, S.; GROCE, N.; JOHNSON, A.; KETT, M.; LEE, M.; LEVY, C.; MASLIN, M.; MCCOY, D.; MCGUIRE, B.; MONTGOMERY, H.; NAPIER, D.; PAGEL, C.; PATEL, J.; OLIVEIRA, J. A. P.; REDCLIFT, N.; REES, H.; ROGGER, D.; SCOTT, J.; STEPHENSON, J.; TWIGG, J.; WOLFF, J.; PATTERSON, C. Managing the health effects of climate change. The Lancet, v. 373, n. 9676, p. 1693-733, 2009.

GARCIA, W. D. Reflexos da globalização na cultura alimentar: considerações sobre as 
mudanças na alimentação urbana. Revista de Nutrição, v. 16, n. 4, p. 483-92, 2003.

FRIEL, S.; DANGOUR, A. D.; GARNETT, T.; LOCK, K.; CHALABI, Z.; ROBERTS, I.; BUTLER, A.; BUTLER, C. D.; WAAGE, J.; MCMICHAEL, A. J.; HAINES, A. Public health benefits of strategies to reduce greenhouse-gas emissions: food and agricultural. The Lancet, v. 374, n. 9706, p. 2016-25, 2009.

REIS GH.; BRANDELERO, F.; PEREIRA, L. S.; AMARAL, A. Q.; TOREGEANI, K.; SILVA, A. A.; VIANA, A.; ZELINSKI, M. Aproveitamento integral de alimentos e suas contribuições para a educação ambiental dentro da comunidade escolar. In: Colóquio Internacional da Rede de pesquisa em Educação Ambiental por Bacia Hidrográfica, 1 Encontro Paranaense De Educação Ambiental. Cascavel: Unioeste 2013.

SILVA, E. B.; SILVA, E. S. Aproveitamento integral de alimentos: avaliação sensorial de bolos com coprodutos da abóbora (Cucurbita moschata, L.). Mossoró: Revista Verde, v. 7, n. 5, p. 121-131, 2012.

GONDIN AM, MOURA MF, DANTAS AF, MEDEIROS LS, SANTOS KM. Composição Centesimal e de minerais em cascas de frutas. Ciência e Tecnologia de Alimentos, v. 25, n. 4, p. 825-7, 2005.

EMPRABA. A cultura da banana. Brasília: Editora Embrapa-SPI. 1997; 9-10.

EMAGAa, T. H.; RONKARTA, S. N.; ROBERT, C.; WATHELETA, B.; PAQUOTA, M. Characterization of pectins extracted from banana peels (Musa AAA) under different conditions using a experimental design. Food Chemistry, v. 108, p. 463-71, 2009.

EMAGAb, T. H.; RONKART, R.C.; ROBERT, C.; WATHELETA, B.; PAQUOTA, M. Dietary fire components and pectin chemical features of peels during ripening in banana and plantain varieties. Bioresource Technology. v. 99, p. 4346-54, 2008.

Agência Nacional de Vigilância Sanitária. Portaria CVS-6/99. Brasília: ANVISA; 1999.

DUTCOSKY, S. D. Análise sensorial de alimentos. 3 ed. Curitiba: Champagnat; 2011. p. 36. 
ISHIMOTO, F. Y.; HARADA, A. I.; BRANCO, I. G.; CONCEIÇÃO, W. A. S.; COUTINHO, M. R. Aproveitamento alternativo da casca do maracujá-amarelo (Passiflora edulis f. var. flavicarpa Deg.) para produção de biscoitos. Revista Ciências Exatas e Naturais, v. 9, n. 2, p. 279- 292, 2007.

MigUel, A. C. A.; AlBeRTINI, S.; BEGIATO, G. F.; DIAS, J. R. P. S.; SPOTO, M. H. S. Aproveitamento agroindustrial de resíduos sólidos provenientes do melão minimamente processado. Revista Ciência e Tecnologia, v. 28, n. 3, p. 733-737, 2008.

BRAGA LV.; MORAES, J. M. D.; FREIRE, L. A. F.; LIMA, N. C. M.; PORTELA, J. O. Avaliação sensorial de docinho elaborado com polpa e casca de banana. In: IX ENPPG, IX ENICIT, III SIMPIT. Instituto Federal de Educação, Ciência e Tecnologia do Ceará. Fortaleza, 2009.

BOBBIO, P. A.; BOBBIO, F. O. Química do processamento de alimentos. 3. ed. São Paulo: Varela; 2001. p.143.

SANTOS, J. M. Desenvolvimento de bananada da casca da banana com redução do valor Calórico. [Trabalho de Conclusão de Curso]. Vitória: Faculdade Católica Salesiana do Espírito Santo; 2014. 90f.

MIRANDA, A. A.; CAIXETA, A. C. A. FLÁVIO, E. F.; PINHO, L. Fibras da farinha da casca de maracujá. Alimentos e Nutrição, v. 24, n. 2, p. 225-32, 2013. 